\title{
Stable Bundles \\ (Commentary on [95])
}

Lisa Jeffrey

The article "Stable Bundles Revisited" is a review article describing the fundamental work of Atiyah and Bott in [1], where the authors prove formulas for the Poincaré polynomial of the moduli space $M(n, d)$ of stable bundles of rank $n$ and degree $d$ over a Riemann surface. Atiyah and Bott are motivated by Morse theory of the normsquare of the moment map for the action of gauge group on the space of all connections on the surface (an infinite-dimensional vector space). Atiyah and Bott made the fundamental observation that the action of the gauge group on the space of all connections is Hamiltonian and the moment map is the curvature.

There were many obstacles to proceeding with Morse theory in this infinite-dimensional situation, as with a finite-dimensional manifold (as in Frances Kirwan's D.Phil. thesis) [3]. In finite dimensions a Morse function provides a stratification of the manifold indexed by the critical points of the function, where each stratum contributes a term involving the index of the Morse function. The equivariant Euler class of the normal bundle has no zero divisors, so the Thom-Gysin sequence decomposes into short exact sequences. This enables one to recover the Poincaré polynomial of the minimum stratum of the Morse function in terms of the contributions of the other strata.

Atiyah and Bott instead complete their program using the Harder-Narasimhan filtration. The Poincaré polynomial of $M_{g}(2,1)$ is as follows:

$$
\frac{\left(1+t^{3}\right)^{2 g}-t^{2 g}(1+t)^{2 g}}{\left(1-t^{2}\right)\left(1-t^{4}\right)}
$$

A Morse-theoretic proof of the formulas for the Poincaré polynomials, along the lines of Atiyah and Bott's original method, was completed by Daskalopoulos [2].

\section{References}

[1] M. Atiyah, R. Bott, The Yang-Mills equations over Riemann surfaces. Phil. Trans. Royal Soc. London A 308 (1983) 523-615.

[2] G. Daskalopoulos, The topology of the space of stable bundles on a compact Riemann surface. J. Diff. Geom. 36 (1992), no. 3, 699-746.

[3] F. Kirwan, Cohomology of Quotients in Symplectic and Algebraic Geometry. Princeton University Press, 1983.

\footnotetext{
L. Jeffrey $(\square)$

Department of Mathematics, University of Toronto, Toronto, ON M5S 2E4, Canada

e-mail: jeffrey@math.toronto.edu
} 